\begin{tabular}{|l|l|l|}
\hline \multicolumn{2}{|c|}{ PublisherInfo } \\
\hline \hline PublisherName & $:$ & BioMed Central \\
\hline \hline PublisherLocation & $:$ & London \\
\hline \hline PublisherImprintName & $:$ & BioMed Central \\
\hline \hline
\end{tabular}

\title{
Regulatory function of anergized T cells
}

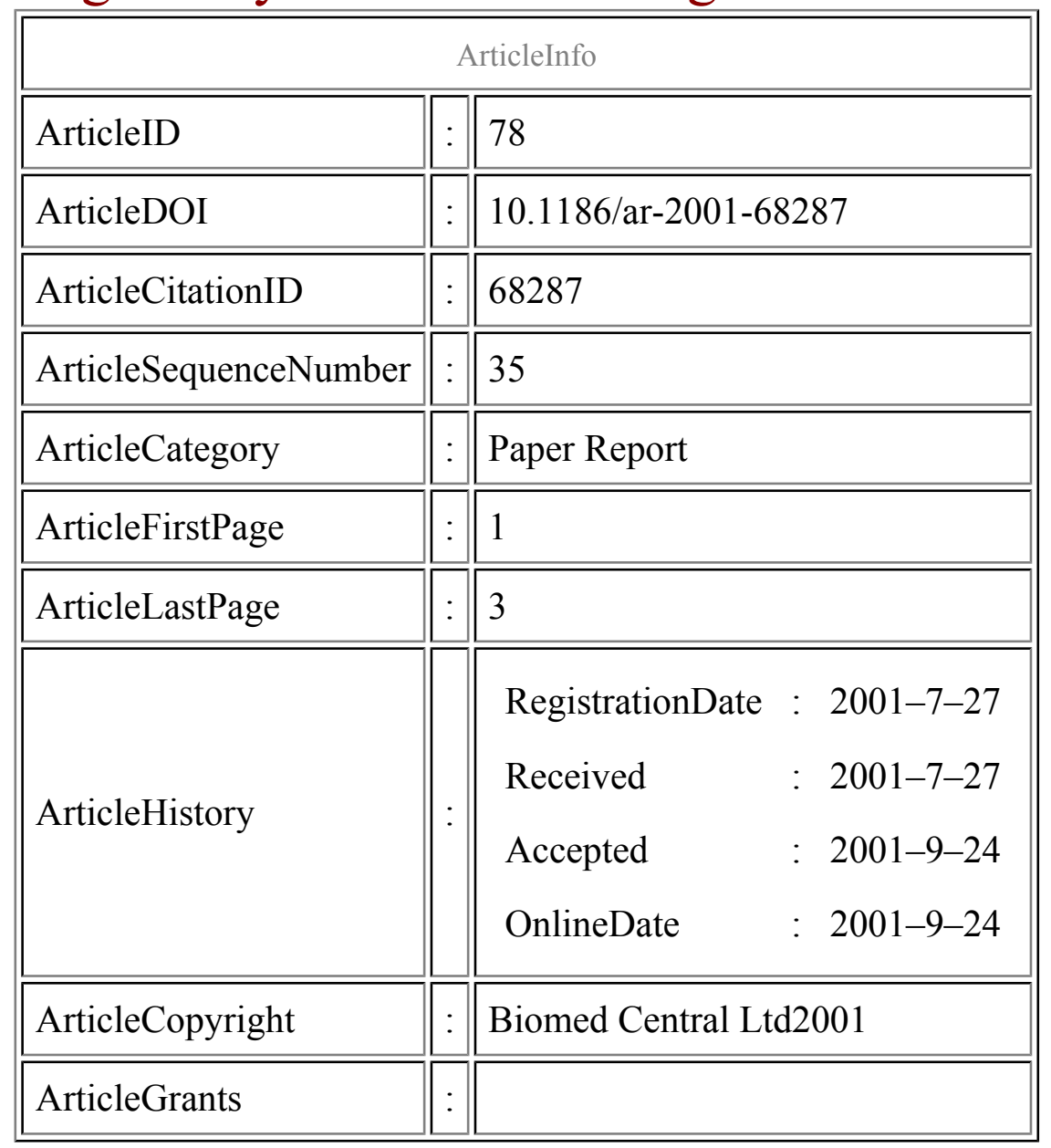




\begin{tabular}{|l|l|l|l|}
\hline ArticleContext & $:$ & 130753311 \\
\hline
\end{tabular}

Steven C Ghivizzani, Aff1

Aff1 Harvard Medical School, Boston, USA

\section{Keywords}

Anergy, gene transfer, immunomodulation, T-cell, tolerance

\section{Context}

Through cross-presentation, antigen-presenting cells (APC) acquire tissue antigens and then either prime or tolerize mature $\mathrm{T}$ cells. The pathway toward either direction is not completely understood, but is thought to involve alternate interactions - of MHC antigens, T-cell receptors and costimulatory molecules - between the two cell types. It has been postulated that anergized T cells arising from this process may not be inert, but instead, actively regulate immune responses; however, this has not been demonstrated in vivo. To study the immunomodulatory capacity of these cells, the authors make use of a novel system whereby anergic $\mathrm{T}$ cells of known antigen specificity are generated in transgenic mice; immune response following local expression of antigen delivered by adenovirus or adeno-associated virus (AAV) vectors are then evaluated.

\section{Significant findings}

Transgenic mice were generated containing the gene for influenza hemaeglutinin (HA) under control of the Ig? light chain promoter and enhancer. This enabled expression of HA on circulating hematopoietic cells. These mice were found to be tolerant of HA expression in muscle following AAV mediated gene transfer. In the same mice, adenoviral mediated expression of HA in muscle was quickly terminated due to a local immune response as evidenced by local muscle fiber destruction. This was attributed to coexpression of vector-encoded native adenoviral proteins. In transgenic mice that expressed a T-cell receptor specific for HA (TCR-HA), HA expressing muscle cells were quickly eliminated by the immune system following delivery with either vector. Anergic $\mathrm{T}$ cells were generated in double transgenic mice that express HA as well as TCR-HA. In these mice, regardless of viral vector used for delivery to muscle, HA expression was found to be stable, Following adoptive transfer into nude mice, anergic $\mathrm{T}$ cells were capable of blocking immune responses of $\mathrm{T}$ cells from TCR-HA mice to either HA or viral antigens of a recombinant adenoviral vector. 


\section{Comments}

The authors have generated a clever experimental system within which to generate and evaluate anergic $\mathrm{T}$ cells in vivo. Their approach enables convincing demonstration that anergized cells remain tolerant of their cognate antigen but are also capable of suppressing the response of naive $T$ cells to irrelevant or bystander antigens, in this case adenoviral antigens, through production of the immunoregulatory cytokine IL-10. This is analogous to infectious tolerance. A limitation of the work is that the mechanisms by which the anergic cells impose this nonrestricted immunosuppression are not experimentally addressed in any depth. The authors suggest several additional candidate molecules, such as CTLA4, PD1 and TGF?, that may be important in directing the activity of these cells, which will be addressed in subsequent experiments. Recently, dendritic cells and antigen-specific $\mathrm{CD} 4^{+} \mathrm{T}$ cells genetically modified to express immunomodulatory proteins IL-4 and IL-12 p40, respectively, have shown promise in treating experimental arthritis in mice. The work in the present study strengthens the concept that autoimmune disease may arise from misdirected APC crosspresentation and that gene therapy strategies designed to specifically enhance populations of circulating anergic/immunoregulatory $\mathrm{T}$ cells could be a particularly potent approach to the treatment of rheumatoid arthritis.

\section{Methods}

Recombinant viral vectors, immunohistochemistry, cytotoxic T lymphocyte assay, adoptive transfer, transgenic mice

\section{Additional information}

\section{References}

1. Jooss K, Gjata B, Danos O, von Boehmer H, Sarukhan A: Regulatory function of in vivo anergized CD4 ${ }^{+}$T cells. Proc Natl Acad Sci USA . 2001, 98: 8738-8743.

This PDF file was created after publication. 